\title{
Barrel tipping in axial piston pumps and motors
}

\author{
Peter Achten, Sjoerd Eggenkamp \\ INNAS BV, Nikkelstr. 15, 4823AE Breda, the Netherlands \\ E-mail: pachten@innas.com, seggenkamp@innas.com
}

\begin{abstract}
In axial piston pumps and motors, the interface between the cylinder block and the port plate is one of the most complicated design details. This interface is a combination of a thrust bearing and a face sealing. The gap height, i.e. the thickness of the oil film, is only allowed to be a few micrometers. A smaller gap height results in high friction losses and possibly in metal-to-metal contact, causing wear and particle generation. On the other hand, a large gap height causes high leakage. In most cases, the barrel or cylinder block will be tipping instead of lifting, thereby causing a variable gap height and combining both high friction losses (where the gap height is small) and high volumetric losses (where the gap height is large). This paper investigates the factors that determine the barrel force and torque balance. Two axial piston pump principles are investigated and compared: a swash plate, slipper type machine and a floating cup pump/motor.
\end{abstract}

Keywords: Axial piston pump, barrel tipping

\section{Introduction}

Positive displacement machines (by definition) have sliding interfaces: machine components sliding on each other, with a thin oil film separating the two components. Some examples are, pistons moving up and down in cylinders, piston slippers running on swash plates and gears running in gear pumps. These interfaces often perform a combination of a bearing and a sealing function. The gap height (i.e. the height of these oil films) needs to be small, but preferably not too small, mostly in the order of a few micrometers. This poses a challenging task for the designer, especially since thermal expansion, pressure deformation and production tolerances can have a substantial effect on the size and geometry of these gaps.

An often-used design principle and solution to this problem is to allow the sliding components to find their own relative position. This solution is used in axial piston machines, for instance, in the sliding interface between the rotating barrel and the stationary port plate, in which the cylinder block is free to find its own position on the port plate (Figure 1). The barrel is connected to the shaft by means of a spline, on which, to some extend, it is allowed to move in the z-direction, and rotate around the $\mathrm{x}$ - and $\mathrm{y}$-axis in point $A$. A spring (not shown in Figure 1) is mounted which pushes the barrel to the surface of the port plate after assembly. Furthermore, the sealing lands of the barrel ports are dimensioned as such that there is a remaining hydrostatic force pushing the barrel even harder to the port plate when the pressure level increases.

When being positioned on the port plate, it seems that the only remaining degree of freedom is the rotation around the $\mathrm{z}$-axis. However, on a micro scale, the oil film still separates the barrel from the port plate, thereby allowing the barrel to move a few micrometers in the z-direction, and rotate or tip around the $\mathrm{x}$ - and $\mathrm{y}$-axis. In the end, these small micro motions determine the volumetric and mechanical losses of the interface between the barrel and the port plate, but also wear, durability and stability of the pump or motor operation.

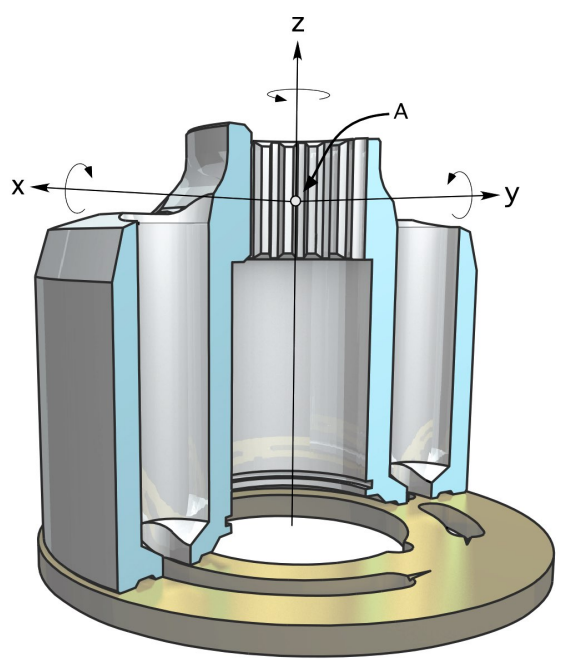

Fig. 1: Barrel and port plate of an axial piston, slipper type machine

In bent axis machines (Figure 2), the tipping around the $\mathrm{x}$ and $y$-axis is often prevented by means of a central pin. This is possible, since bent axis machines don't have a through drive; the drive shaft stops at the drive flange. Moreover, the large tilt angle of the barrel creates a machine having a rela- 
tively long piston stroke and a small barrel diameter. The small diameter of the barrel and the barrel ports is important since the pin reduces the demand for perpendicularity to the surface of the port plate.

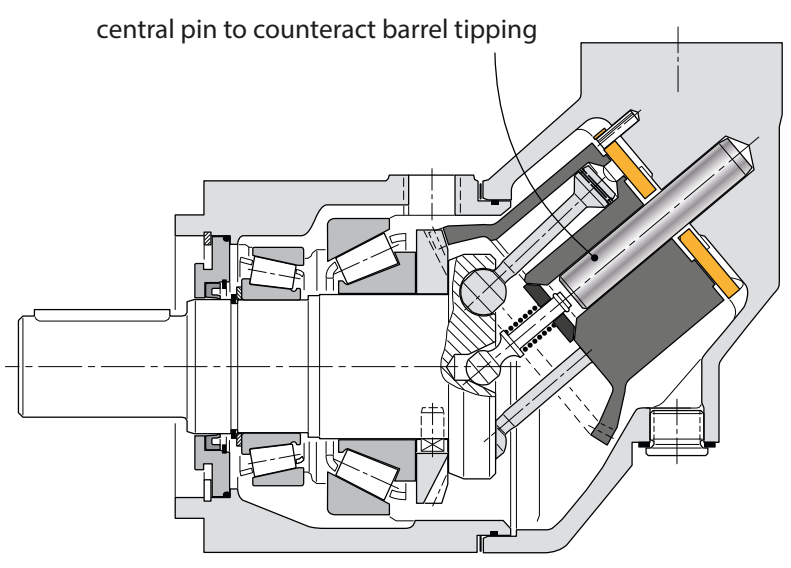

Fig. 2: Cross section of a bent axis pump or motor

Slipper type pumps and motors (Figure 2) have a shaft going through the entire length of the machine. This has the advantage that a second machine can be mounted on the back of the first, but it eliminates the opportunity to apply a central barrel pin. Furthermore, due to the limited swash angle (which is about half of the tilt angle of bent axis machines) the pistons have a shorter stroke and the barrel diameter is larger, which makes it harder to accomplish the perpendicularity between the pin and the sealing area of the barrel ports. As a consequence, the solution of a central barrel pin is not used in slipper type machines, and the barrel is free to tip around the $\mathrm{x}$ - and $\mathrm{y}$-axis shown in Fig. 1.

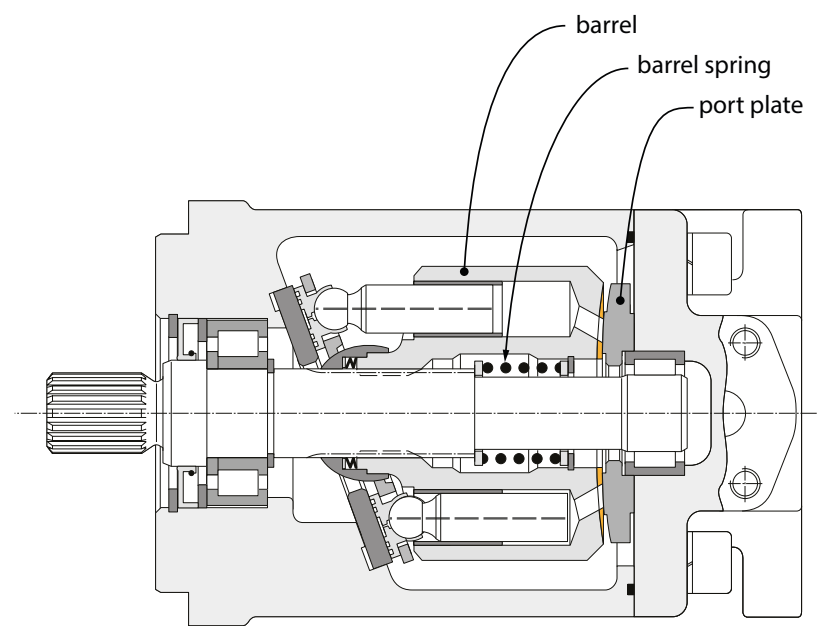

Fig. 3: Cross section of a slipper type pump or motor

Floating cup pumps and motors (Figure 4) have a different construction, in which the pistons are press-fitted into the rotor. Instead, the barrel cylinders have been isolated from the barrel. Each piston is combined with a cup-like cylinder which is floating on the barrel plate. Like in the bent axis design, the barrel has a tilted position relative to the main axis. But the tilt angle is much smaller than in bent axis ma- chines, and even smaller than of slipper type machines. The small tilt angle results in a multi-piston design, having two rings of pistons, arranged in a back-to-back configuration.

Like in slipper type machines, the shaft is going through the entire length of the machine. Another similarity with the slipper type machine is the relatively large diameter of the barrels. The barrels are allowed to rotate around the pivot points, indicated with $A$ in Figure 4.

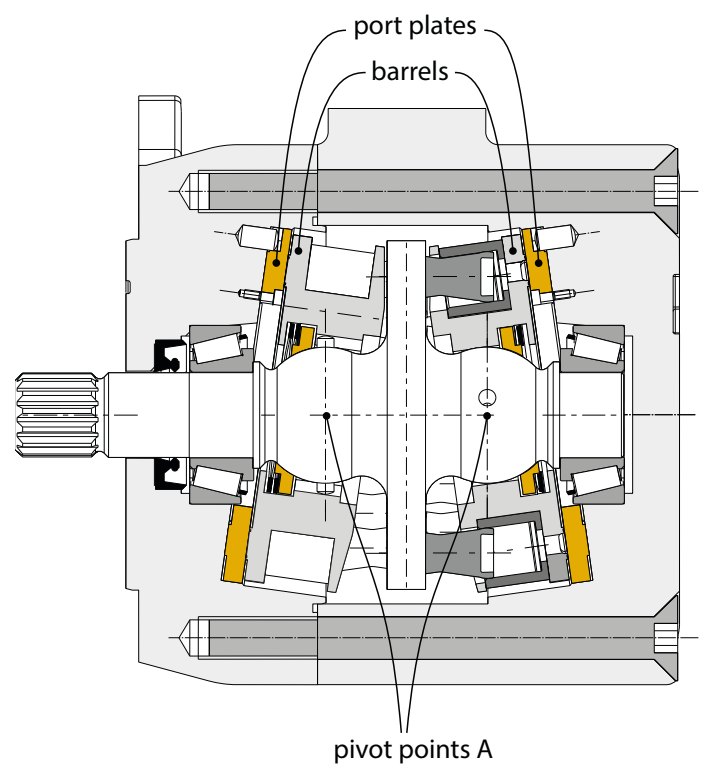

Fig. 4: Cross section of a floating cup pump or motor

The floating cup design belongs to the family of axial piston principles: the barrels are free to find its position on the port plates, thereby being positioned after assembly by a barrel spring. And, just like in other axial piston designs, the force and torque balancing is a difficult design challenge.

This paper discusses the factors that influence the tipping of the barrel in axial piston machines, in particular the amount of spring force which is needed to avoid barrel tipping at high revolutionary speeds and low operating pressures. The paper will only discuss slipper type and floating cup pumps and motors.

\section{Tipping torque research}

There are many different loads acting on the barrel, which result in a torque load around the $\mathrm{x}$ - and $\mathrm{y}$-axis of the barrel:

- Hydrostatic pressure forces inside the cylinder;

- Hydrostatic pressure forces acting on the barrel ports and sealing lands;

- Centrifugal forces;

- Friction forces between the pistons and the cylinders;

- Friction between the barrel and the port plate;

- Lateral piston forces and torques;

- Force of the axial barrel spring;

- Impulse forces of the oil flow.

The hydrostatic forces from the sealing lands are dependent on the pressure profile in the gap between the barrel and the 
port plate. The pressure profile is strongly influenced by elasto-hydrodynamic and thermal deformations, as well as by the variation of the viscosity of the oil, while passing the gap, due to temperature and pressure variations [1-4]. The gap profile is also influenced by the precise barrel position on the valve plate, and hence by the tipping itself.

Many of the underlying physical phenomena are, up to now, still not completely understood. The first modeling attempts started in the early 80's [5-7]. These first models were based on rigid-body models $[8,9]$. By the turn of the century, the increased capacity and performance of computers allowed the addition of elasto-hydrodynamic (EHD) effects [10-12]. At first, only the deformation of the barrel and the port plate was calculated. In 2006 [13], it became clear that the deformation of the entire housing strongly affected the bearing interface between the barrel and the port plate. Most recent simulations also include the thermal expansion and deformation of the components $[3,14,15]$, and the non-flatness and micro-geometry of the bearing surfaces $[16,17]$. The calculation of the dynamic micro-motion of the cylinder block has become a complex, multi-domain simulation effort, combining EHD with CFD-analysis, thermal analysis and multi-body mechanical analysis. Yet, despite this enormous progress in simulation techniques, the theoretical analysis does not yet include all relevant aspects, like production tolerances, surface roughness and wear.

Aside from the analytical complexity, it is almost impossible to get a direct, experimental verification of the analytical results. Often, separate test benches are built, in which components, like the cylinder block, the pistons and the port plate, are isolated from the rest of the pump. Figure 5 shows an example of such a test bench, as was developed by Bräckelmann [18, 19]. Consequently, important effects, like the deformation of the housing, are neglected. Other factors, like the bending of the main shaft, are often enlarged, since a special shaft is often constructed for these benches.
Yamaguchi $[5,6]$ was one of the first to measure the gap height and tipping of a barrel-like structure running on a valve plate. Other experimental research in this area has been performed by Ivantysynova and her team [3, 4, 14-16, 20], Bergada, et al [21-23], Wegner, et al [24, 25], Shin, et al [17], Han, et al [26] and Zhang, et al [27]. Most of these studies measured a gap height between 0 and $20 \mu \mathrm{m}$. The experiments also showed a tilted barrel position, creating a wedge shaped fluid film with a varying gap height.

Extensive measurements were performed by Bräckelmann [18] on a relatively small 40 cc swash plate pump. The gap height was measured to be 2 to $14 \mu \mathrm{m}$, depending on the rotational speed and the oil pressure. Furthermore, Bräckelmann measured a tipping of the barrel of up to $0.07^{\circ}$. The tipping angle proved to be about linearly dependent of the rotational speed. Furthermore, a larger swash angle and a higher pressure level also increased the barrel tipping.

The influence of the rotational speed on the barrel tipping was also investigated by Noah Manring [28-30]. According to Manring, 'Tipping the cylinder block within an axial-piston swash-plate type hydrostatic machine is a phenomenon that results in a momentary and sometimes permanent failure of the machine' In his paper from 2000 [29] he presented a design criterion for the required force of the central spring of the cylinder block:

$F_{s p}>\frac{N \cdot M \cdot r^{2} \cdot \omega_{\max }^{2} \cdot \tan (\alpha) \cdot\left(1+\tan ^{2}(\alpha)\right)}{2 R}$

in which $F_{s p}$ is the spring force, $N$ is the number of pistons, $M$ is the mass of piston and slipper, $\omega_{\max }$ is the maximum rotational speed, $r$ is the piston pitch radius, $R$ is the outermost radial point of contact on the cylinder block, and $\alpha$ is the swash plate angle.

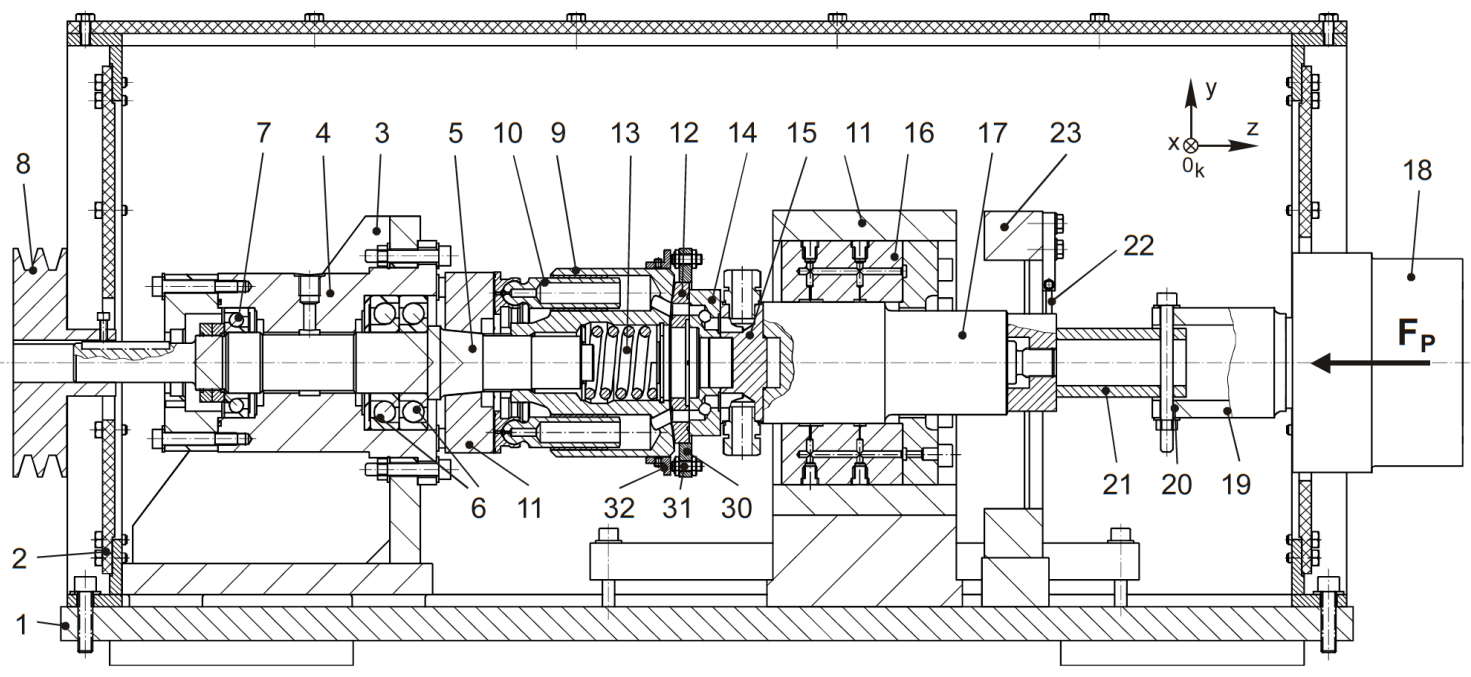

Fig. 5: Test bench for measuring the interface between the barrel and port plate of a swash plate pump [18] 
In the paper from 2014 [30], Manring, et al, adapted this equation:

$$
\omega^{2}<\frac{2 \cdot F_{s p} \cdot R}{N\left(M_{p}+M_{s}\right) r^{2} \cdot \tan (\alpha)}
$$

$M_{p}$ and $M_{s}$ are the mass of the piston, respectively of the slipper. This equation can be rewritten as:

$$
F_{s p}>\frac{N \cdot M \cdot r^{2} \cdot \omega_{\max }^{2} \cdot \tan (\alpha)}{2 R}
$$

which is essentially different from his earlier criterion (equation (1)). Manring does not offer an explanation for this difference. Manring also simplifies the reaction loads from the pistons on the cylinder block. As a result of the angled position of the slipper on the swash plate, the slipper creates a lateral load on the piston. This load results in a torque load on the cylinder block. Manring seems to ignore this torque load. He also neglects the friction between the pistons and the cylinders, which also create a tipping torque on the barrel. Finally, he does not include the friction between the barrel and the valve plate and the friction between the slippers and the swash plate. Due to the variation of the thickness of the oil film in between the barrel and the valve plate, the friction force will also vary around the circumference of the barrel sealing lands, which will cause another tipping torque. Also, the fluid film underneath the slippers varies in height.

This paper presents a new analysis of the torque loads acting on the cylinder block. The aim of the study is to determine the axial force of the cylinder block that is required to prevent the barrel from tipping. Instead of detailed deformation analysis and CFD-calculations of the bearing gaps, simple friction models will be used with constant friction coefficients. Following Manring's analysis, the analysis will be based on a simple rigid-body analysis, and will therefore not include any elasto-hydrodynamic deformations, thermal expansions, or detailed CFD-analysis. However, unlike Manring's analysis, this study also includes the torque loads of the pistons on the barrel, as well as the friction between the pistons and the cylinders.

Two machines will be considered and compared:

- A swash plate type constant displacement pump/motor from Bosch Rexroth (A4FO28 or A4FM28);

- A floating cup constant displacement pump/motor from INNAS.

Both machines have a geometric displacement of $28 \mathrm{cc}$ per revolution.

\section{Swash plate pump or motor}

Figure 6 shows the pistons, the barrel and the barrel spring as isolated components, including some of the loads acting on these components. Only two pistons are shown, whereas in reality most swash plate machines have nine pistons. Figure 6 is also a simplification since all vectors are only shown in a two-dimensional representation, whereas, some of these forces also have a component in the third, missing dimension.

A model has been developed in which all these forces and components are combined. The model does not include the slipper friction $F_{s f}$. According to Bräckelmann [18], this force is negligible compared to the hydrostatic lateral piston load.

In swash plate machines, the pistons are severely loaded due to the kinematic principle. The high lateral loads in the contact between the pistons and the cylinders cause substantial friction loads on the barrel [31].

The dimensions and design parameters of the A4FM28/A4FO28 have been determined by means of disassembling an existing machine and measurement of the dimensions, weights and other relevant parameters (Table 1). The force of the barrel spring has been measured. The data about the friction between the barrel and the valve plate are taken from the thesis of Bräckelmann [18]. The commutation in the top and bottom dead centers is assumed to be instantaneous.

Table 1: A4FM28/A4FO28 parameters

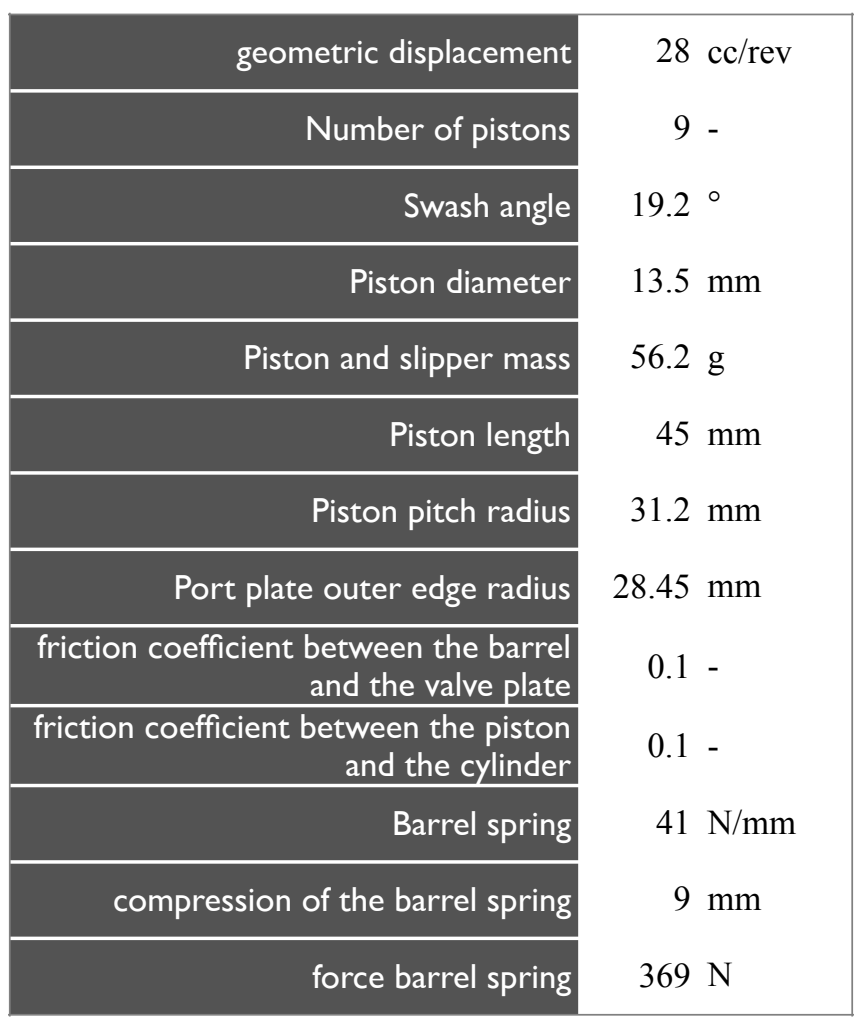




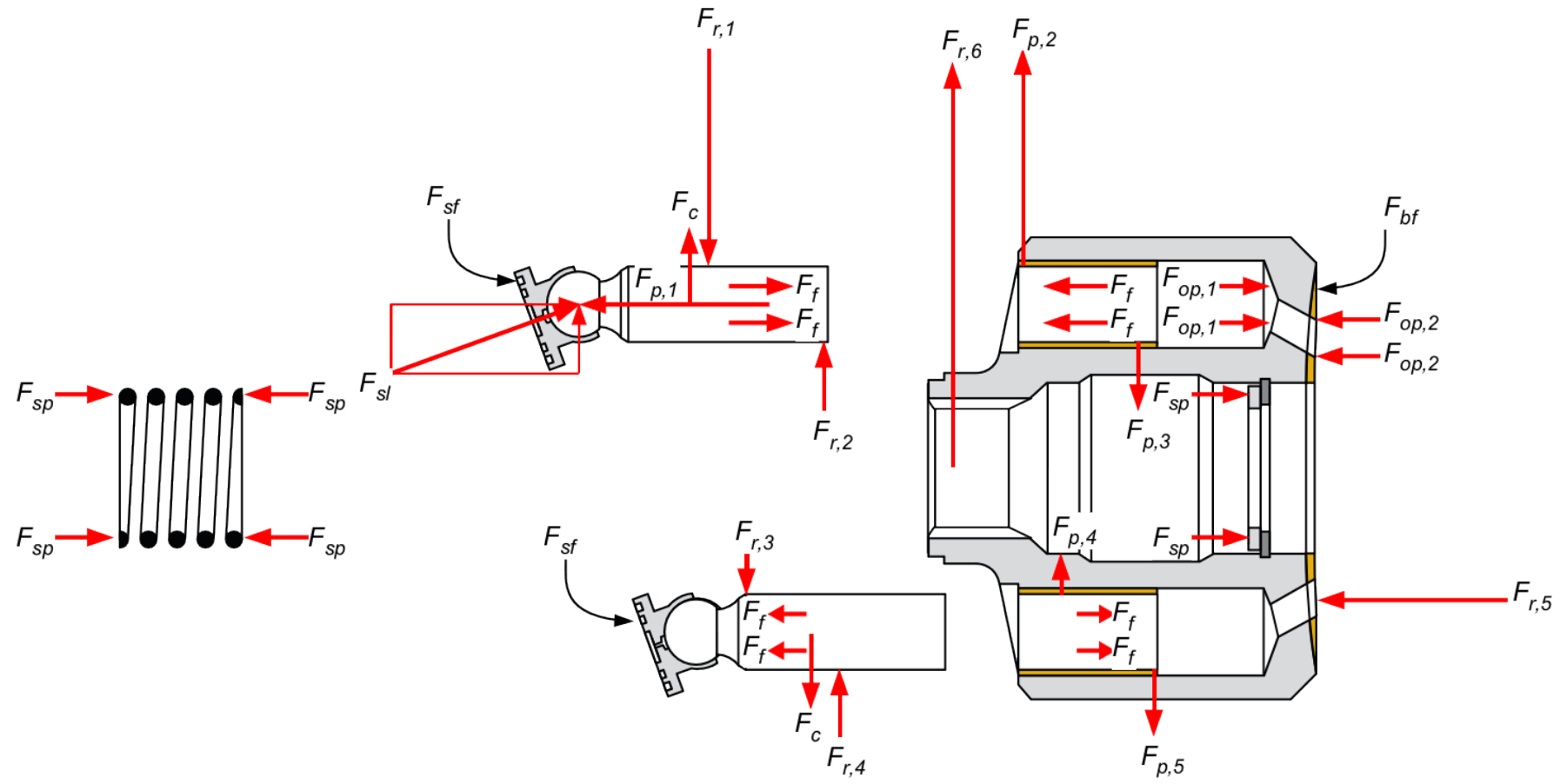

Fig. 6: Loads acting on the pistons, the cylinder block and the barrel spring of a swash plate type pump or motor, showing only 2 pistons, assuming only a pressure load on the upper piston

$F_{p}$ hydrostatic force acting on the piston

$F_{s l}$ Hydrostatic load of the slipper

$F_{c}$ Centrifugal force of the piston and slipper

$F_{f}$ Friction force between the piston and the cylinder

$F_{s f}$ Friction between the slipper and the swash plate

$F_{s b}$ Friction between the barrel and the valve plate

$F_{r, 1}, F_{r, 2}, F_{r, 3}, F_{r, 4}$ Reaction force, resulting from the contact between the piston and the cylinder, acting on the piston

$F_{p, 1}, F_{p, 2}, F_{p, 3}, F_{p, 4}$ Reaction force, resulting from the contact between the piston and the cylinder, acting on the cylinder

$F_{o p, 1}$ Hydrostatic force acting on the bottom of the hydraulic cylinder

$F_{o p, 2}$ Hydrostatic force acting on the barrel ports and sealing lands

$F_{s p}$ Force of the central barrel spring

$F_{r, 5}$ Reaction force between the barrel and the port plate to counteract the tipping torque

$F_{r, 6}$ Sum of all radial piston loads acting on the spline of the barrel

The parameters from Table 1 can be substituted in Equation 2 to calculate the maximum rotational speed before, according to Manring, et al [30], the cylinder block starts to tip:

$$
n_{\max }=3342 \mathrm{rpm}
$$

Equation 2, however, does not contain the pump pressure as a parameter influencing the tipping torque. But, it is certain that a higher pump pressure creates a higher torque load of the pistons on the cylinders of the cylinder block, and, in addition, a higher friction torque.
In order to include the pressure load, a new simulation model has been made, which includes all the loads shown in Figure 6. The coordinate system for these calculations is defined in figure 7 . The torque load acting on the barrel will make the reaction force move out of the centre. If this force moves outside the circle with radius $\mathrm{R}$, the barrel will tip. 


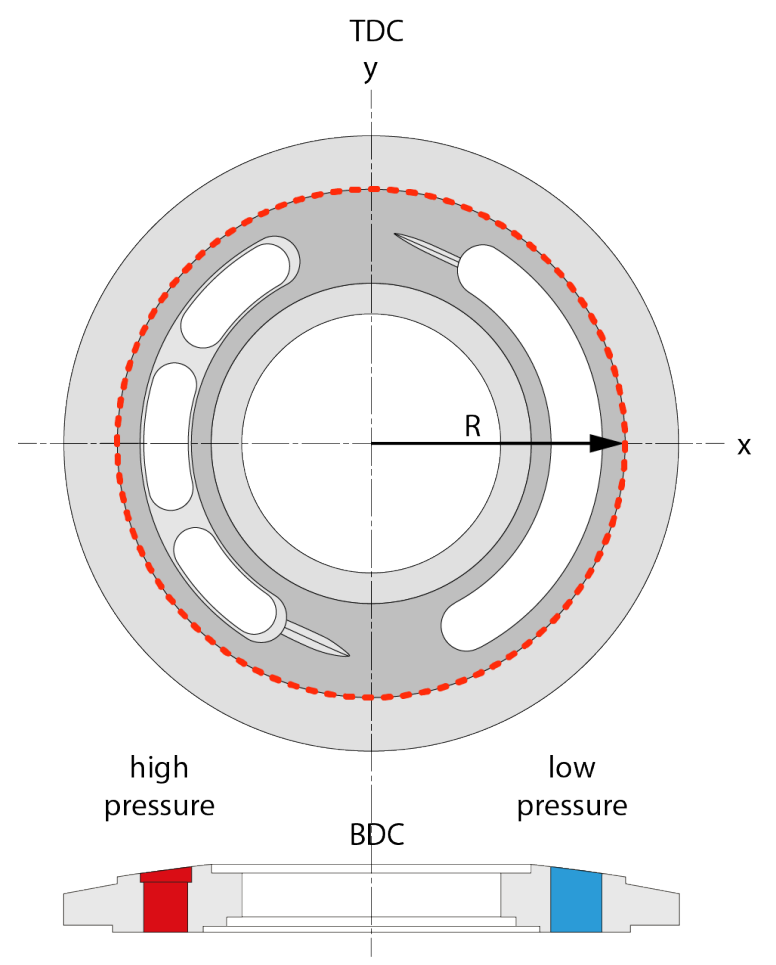

Fig. 7: Top view and cross section of the valve plate of the A4FO28-pump. The red dotted line shows the outmost radius to support the cylinder block against tipping.

In a first calculation, the hydrostatic axial force balance is assumed to be $100 \%$ : the size of the seal lands is chosen as such that the counteracting hydrostatic force is exactly equal to the hydrostatic force, which is pushing the barrel towards the valve plate. In this theoretical situation, only the barrel spring is pushing the cylinder block to the valve plate. The calculated position of the reaction force is displayed in Figure 8, for two different operating speeds and four different pump pressures.

The simulation results (Figure 8a) show the strong influence of the pump pressure on the tipping torque. Actually, the barrel will tip at almost all operating conditions. Only for low operating pressures, below 100 bar, the pump can be operated up till about $3000 \mathrm{rpm}$, without tipping of the barrel.

In a second simulation, the hydrostatic, axial balance has been changed to $93 \%$. In other words, $7 \%$ is no longer compensated by the hydrostatic pressure field in between the barrel and the port plate. This corresponds with the actual dimensions of the components of the A4FO28.

This analysis (Figure 8b) shows that a hydrostatic force is needed to prevent the barrel from tipping. At a pressure level of 400 bar, this hydrostatic barrel force has an average value of $1850 \mathrm{~N}$. The strong spring force is needed to prevent the barrel from tipping at low pump pressures in combination with a high rotational speed.

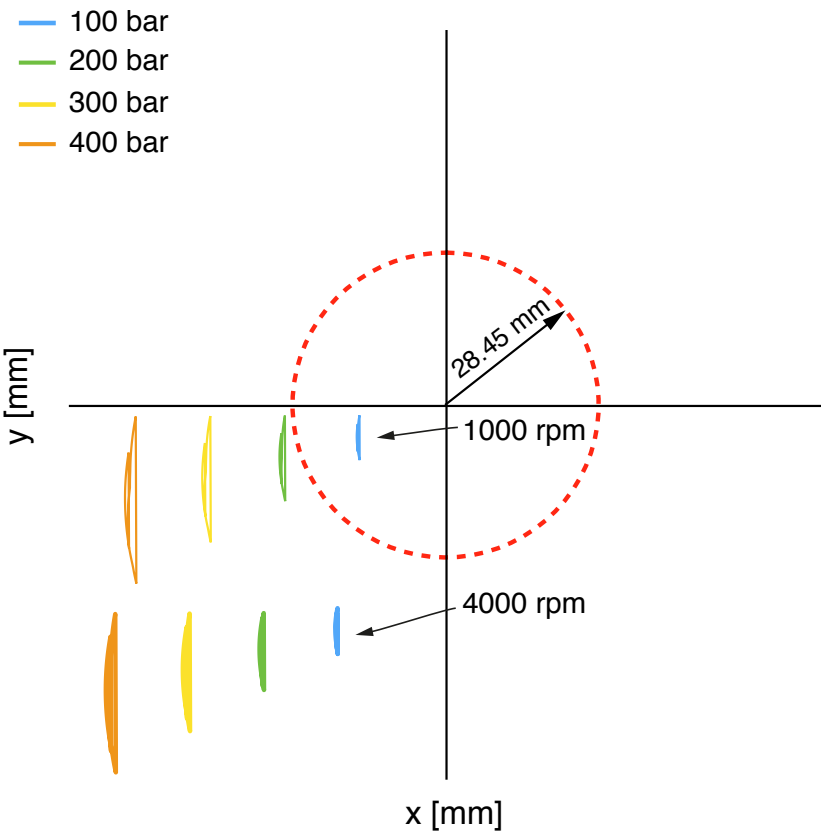

a) Calculated for a hydrostatic axial force balance of 100\%

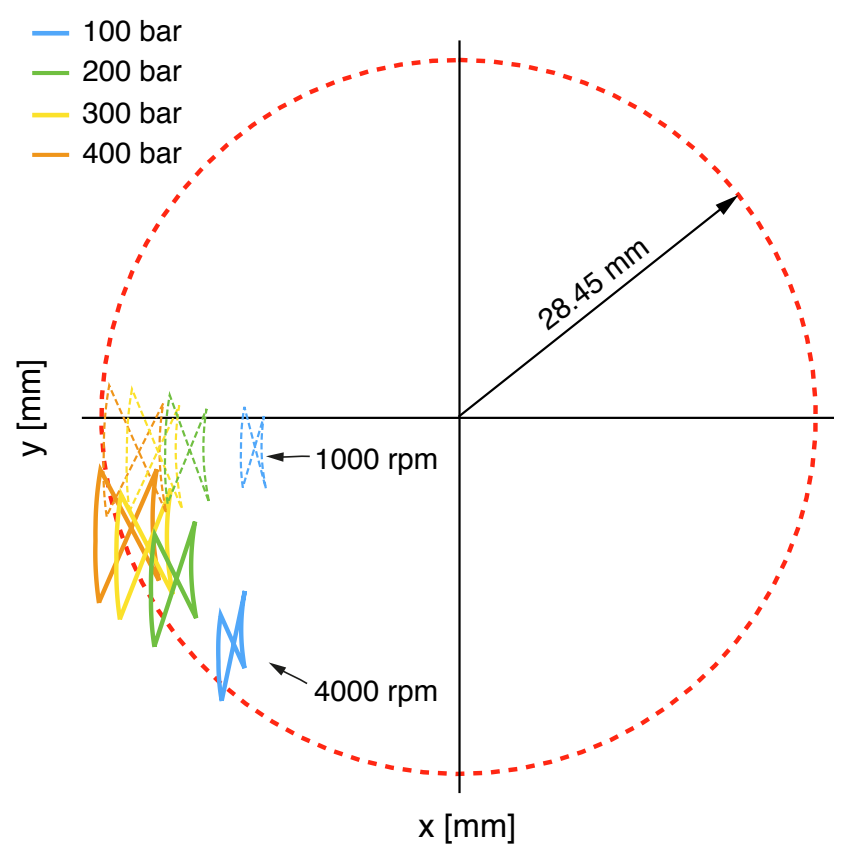

b) Calculated for a hydrostatic axial force balance of 93\%

Fig. 8: Position of the axial reaction force, necessary to counteract the tipping torque, calculated for te slipper type machine for two different hydrostatic balances. The dotted red circle is the outer edge of the port plate to support the barrel. If the reaction force is outside this circle, the barrel will tip. 


\section{Floating cup pump or motor}

A floating cup machine is essentially different from a bent axis or a slipper type pump or motor. Figure 10 shows a cross section of the main rotating parts. Figure 11 shows the main loads acting on the barrel. Table 2 gives the main parameters of the $28 \mathrm{cc}$ floating cup pump or motor.

Table 2: 28 cc FC-pump or motor parameters

\begin{tabular}{|c|c|}
\hline geometric displacement & $28 \mathrm{cc} / \mathrm{rev}$ \\
\hline Number of pistons & $24-$ \\
\hline Swash angle & $8^{\circ}$ \\
\hline Piston diameter & $12.5 \mathrm{~mm}$ \\
\hline Piston and slipper mass & $5.6 \mathrm{~g}$ \\
\hline Piston pitch radius & $34 \mathrm{~mm}$ \\
\hline Port plate outer edge radius & $34.28 \mathrm{~mm}$ \\
\hline $\begin{array}{r}\text { friction coefficient between the barrel } \\
\text { and the valve plate }\end{array}$ & $0.1-$ \\
\hline $\begin{array}{r}\text { friction coefficient between the piston } \\
\text { and the cylinder }\end{array}$ & $0.1-$ \\
\hline force barrel spring & $100 \mathrm{~N}$ \\
\hline
\end{tabular}

In floating cup machines, the pistons are press-fitted into the rotor, and can therefore not create a centrifugal load on the barrel. Instead, the cups are free to move and rotate around the piston crown. The position of the centre of gravity of the cup mostly differs from the midpoint of the piston crown, and consequently the cups will create a centrifugal torque load on the barrel. This torque load is counteracted by the forces $F_{r, 1}$ and $F_{r, 2}$. The number of cups per barrel (12 in this case) is larger than the number of pistons in most axial piston machines. Also the piston pitch radius is somewhat larger. Both factors increase the centrifugal load on the barrel. On the other hand, the weight of the cups is much smaller, about $10 \%$ of the piston of the axial piston pump. Also the stroke length is much smaller. In total, the centrifugal load on the barrel in the floating cup machine is $93 \%$ smaller than in the slipper type machine.

Another difference concerns the piston friction. Due to the hydrostatic balanced design of the cup, there is almost no friction between the pistons and the cups [32]. The only friction is caused by the centrifugal force of the cup and its oil contents. This (small) friction force has been included in the calculation of the barrel tipping torque.

The hydrostatic balance of the barrel is also essentially different. A new hydrostatic bearing is applied in the floating cup machine [33]. The new hydrostatic bearing creates a variable, gap height dependent pressure load in the sealing lands of each barrel port. In principle it creates a perfect axial hydrostatic balance. Only the barrel spring force re- mains to push the barrel towards the port plate. Compared to the slipper type pump, the barrel spring force relatively low, being about $27 \%$ of the spring force in the slipper type pump. This force is also counteracted by the new hydrostatic bearing.

The cups make a small relative movement on the barrel plate [34]. Consequently, the position of the hydrostatic cup force also moves relative to the compensating hydrostatic force of the barrel port and the corresponding seal lands. This results in an additional torque load on the barrel, which is pressure dependent.

Figure 9 shows the calculated position of the resulting barrel force, which is needed to counteract the tipping torque of the barrel. The force trajectories are calculated for two different rotational speeds and 4 different pressure levels. The calculation is performed assuming a constant pressure in the pockets of the seal lands [33]. In reality, the pressure in the pockets varies depending on the local gap height. As a result, the pockets create an additional torque load on the barrel, which counteracts the barrel tipping.

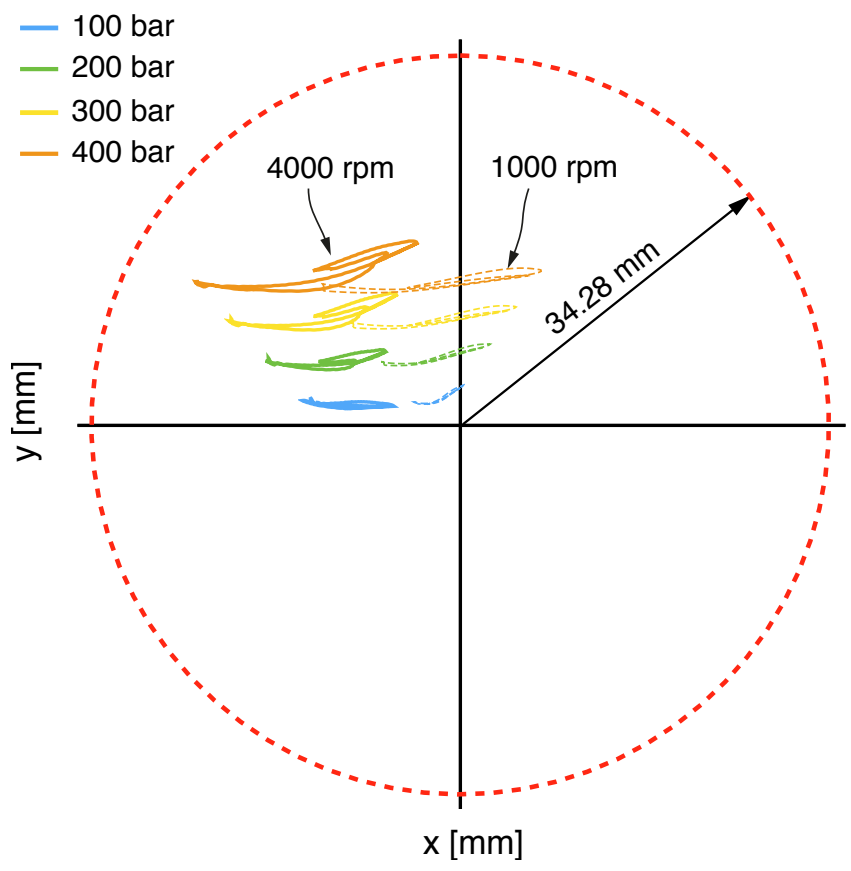

Fig. 9: Position of the axial reaction force, necessary to counteract the tipping torque, calculated for the 28 cc floating cup pump or motor. The dotted red circle is the outer edge of the barrel sealing lands to support the barrel. If the reaction force is outside this circle, the barrel will tip.

The calculation shows that the barrel in the floating cup machine does not tip at $4000 \mathrm{rpm}$, even at high operating pressures. All curves stay within the outer diameter of the largest seal land. 


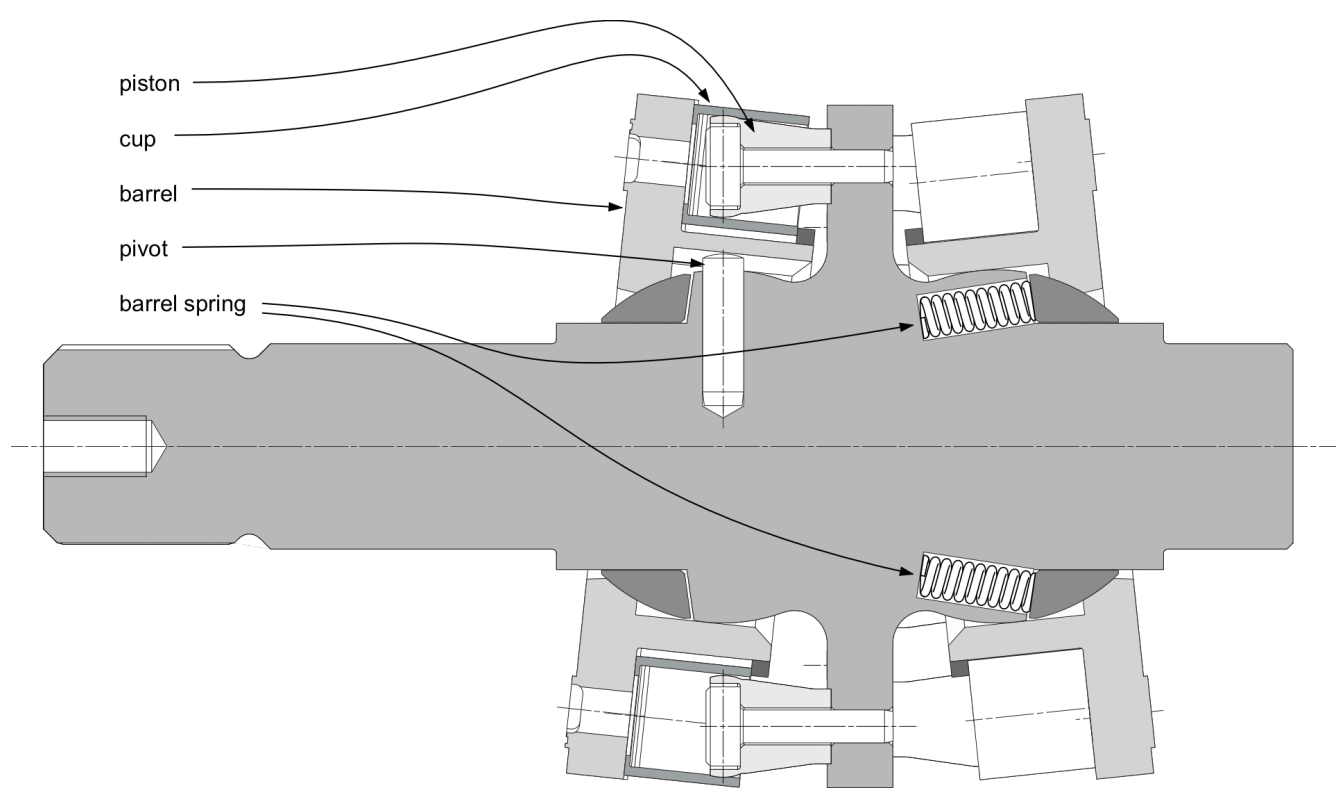

Fig. 10: Cross section of the rotating parts of a floating cup pump

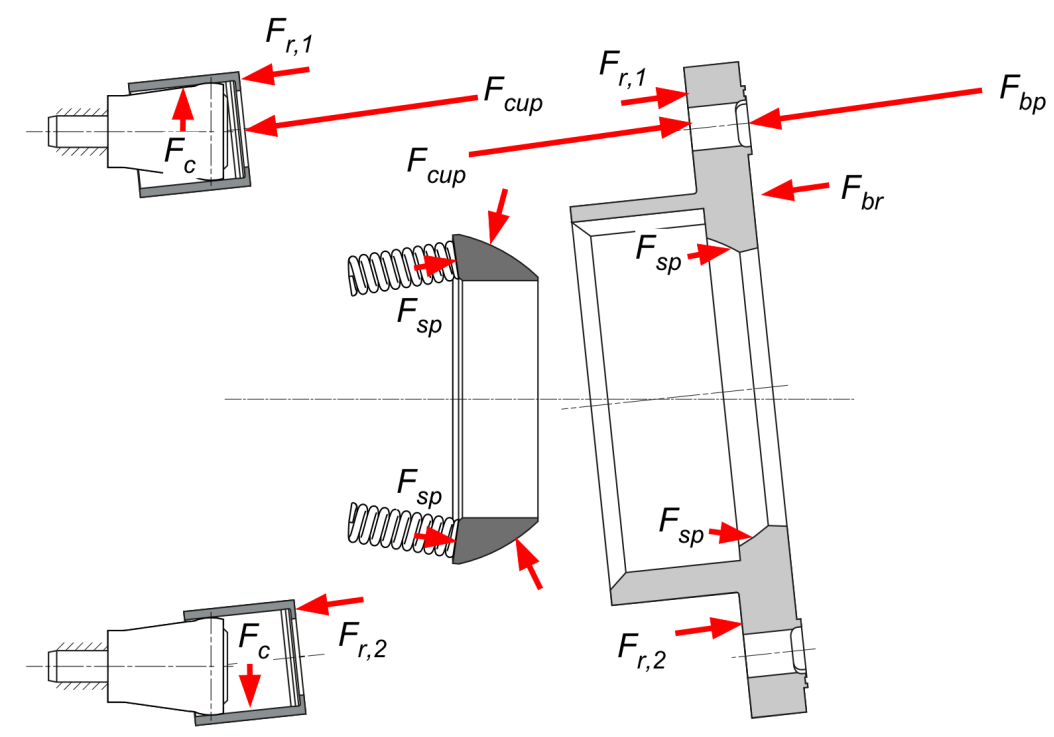

Fig. 11: Loads acting on the cups, the cylinder block and the barrel spring of a floating cup pump or motor, showing only 2 pistons, assuming only a pressure load on the upper piston

$F_{c u p}$ axial hydrostatic force created by the cup $F_{c}$ Centrifugal force of a cup

$F_{r, 1}, F_{r, 2}$ reaction force to counteract the centrifugal torque of the cup $F_{s p}$ Force of the barrel spring

$F_{b p}$ hydrostatic force create by the barrel port and seal lands

$F_{b r}$ Reaction force of the barrel to counteract the tipping torque 


\section{Comparison of the tipping torque}

The simulations show that the tipping torque is strongly influenced by the design principle. For both design principles, the total tipping torque load on the barrel is given in Table 3, as is calculated for 16 different operating conditions. On average, the tipping torque of the floating cup design is only $3.3 \%$ of the barrel tipping torque of a slipper type design. The strong reduction is the result of a combination of improvements. Most important are, the reduction of the centrifugal forces by about $93 \%$ and the elimination of the lateral loads between the pistons and the cylinders.

Table 3: Calculated average tipping torque in $\mathrm{Nm}$ for a 28 cc slipper type (ST) and floating cup machine (FC)

\begin{tabular}{|l|l|l|l|l|l|l|l|l|}
\hline \multirow{2}{*}{ p [bar] } & \multicolumn{2}{|c|}{$1000 \mathrm{rpm}$} & \multicolumn{2}{|c|}{$2000 \mathrm{rpm}$} & \multicolumn{2}{|c|}{$3000 \mathrm{rpm}$} & \multicolumn{2}{|c|}{$4000 \mathrm{rpm}$} \\
\cline { 2 - 10 } & ST & FC & ST & FC & ST & FC & ST & FC \\
\hline $\mathbf{1 0 0}$ bar & 14.3 & 0.37 & 15.2 & 0.43 & 17.6 & 0.70 & 22.1 & 1.21 \\
\hline $\mathbf{2 0 0}$ bar & 28.7 & 0.75 & 29.3 & 0.84 & 31.0 & 1.06 & 34.3 & 1.48 \\
\hline $\mathbf{3 0 0}$ bar & 43.0 & 1.13 & 43.6 & 1.22 & 45.0 & 1.41 & 47.8 & 1.78 \\
\hline $\mathbf{4 0 0}$ & 57.4 & 1.50 & 57.9 & 1.59 & 59.2 & 1.77 & 61.6 & 2.11 \\
\hline
\end{tabular}

\section{Contact force between the barrel and the valve plate}

The slipper type pump needs a strong axial barrel force to keep the barrel from tipping. Even a relatively strong barrel spring is not sufficient to avoid barrel tipping (see Figure 9a). Only when adding a substantial hydrostatic force, the unit can be operated to rotational speeds of about $3000 \mathrm{rpm}$.

For this last situation, the calculated contact force between the barrel and the valve plate is displayed in the diagram of Figure 12. The diagram shows the calculated contact force for four different pressure levels. Due to the simplified model of the instantaneous commutation, the calculated force is not influenced by the rotational speed.

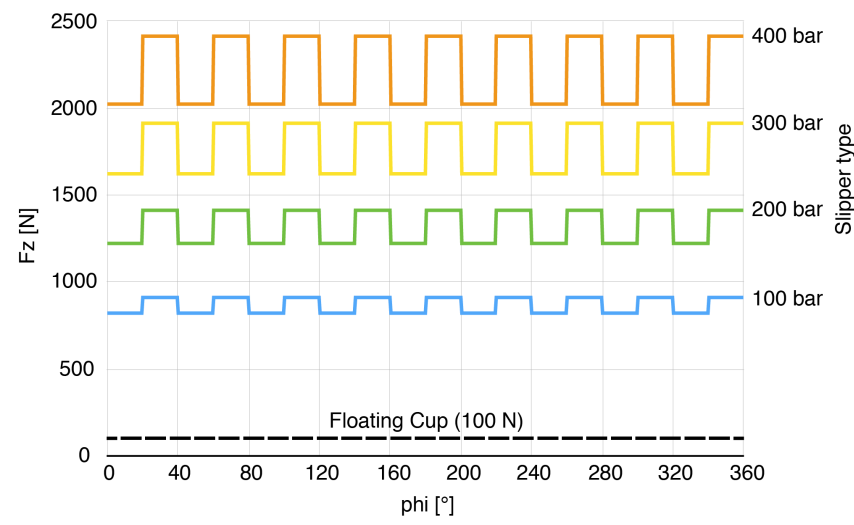

Fig. 12: Calculated contact force between the barrel and the valve plate
The calculation does not include any hydrodynamic effects, nor any elasto-hydrodynamic-deformation which could influence the gap geometry and the bearing capacity. The calculated force is therefore not necessarily a contact force but can be regarded as the force that needs to be overcome by for instance hydrodynamic effects.

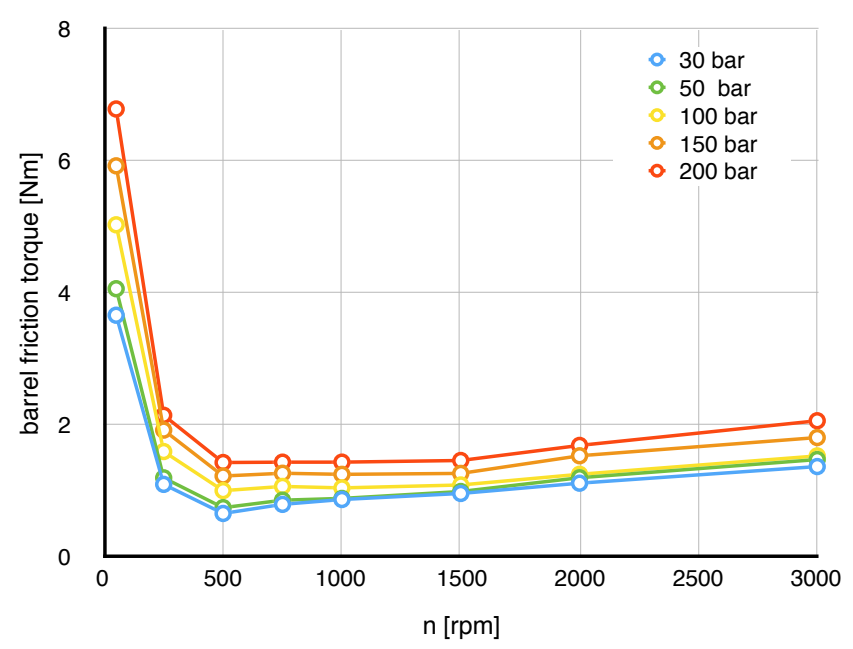

Fig. 13: Friction torque between the barrel and the port plate, measured for a $56 \mathrm{cc}$ slipper type machine

The hydrodynamic lubrication is demonstrated by Bräckelmann [18] by building a separate test bench to measure the friction torque at various operating conditions (Figure 5). Figure 13 shows the measured friction torque for a $56 \mathrm{cc}$ slipper type machine. The measurements are consistent with the Stribeck-effect, showing mixed lubrication conditions and high friction at low operating speeds. For operating speeds of $500 \mathrm{rpm}$ and higher, the friction losses are more or less linear with the operating speed. Nevertheless, the operating pressure creates an additional friction torque, indicating coulomb friction at these conditions.

In the floating cup machine, the axial load between the barrel and the port plate is almost negligible compared to the slipper type. As a consequence, the floating cup principle does not have as much friction losses between the barrel and the valve plate, especially at startup and low operating speeds.

\section{Conclusions}

In axial piston machines, the operating conditions can be constrained because of tipping of the cylinder block or barrel. Manring $[29,30]$ has already analysed the relationship between operating speed and barrel tipping. However, in these publications, the torque balance on the cylinder block is strongly simplified, and does not include pressure dependent loads.

This paper has shown, that the operating pressure has a stronger influence on the barrel tipping than the operating speed. In order to avoid tipping in slipper type machines, the barrel and port plate geometry needs to be dimensioned as such that the barrel is not completely balanced in the axial direction. Of the total hydrostatic force exerted by the axial hydrostatic force, only $93 \%$ can be compensated by the bar- 
rel ports and seal lands. The remaining $7 \%$ is needed to sufficiently press the barrel against the port plate, thereby counteracting the tipping torque created by the pistons. Nevertheless, despite the strong axial force, the maximum rotational speed is constraint by the high centrifugal forces and the strong tipping torque created by the pistons.

In this respect, the floating cup principle performs much better. The centrifugal load is decimated due to the low cup mass and the short stroke. Furthermore there are hardly any other loads created by the piston-cup-interface. The total tipping torque is reduced by about $97 \%$ compared to the slipper type machine. The force of the central barrel spring can be reduced by more than $70 \%$. Nonetheless, floating cup pumps and motors can be operated at higher operating speeds and pressures without any barrel tipping.

This paper also shows that, in slipper type machines, barrel tipping is related to the efficiency of the pump or motor. In order to prevent barrel tipping, the barrel needs to be pushed stronger in the direction of the valve plate, which increases the friction and reduces the efficiency. In the floating cup principle, this can be avoided completely. The reduced axial force load and tipping torque results in a strong reduction of the friction losses between the cylinder block and the port plate. This results in an increased efficiency and higher startup torque.

\section{References}

1. Pelosi, M. and M. Ivantysynova, The influence of pressure and thermal deformation on the piston/cylinder interface film thickness. In: Proceedings of the 52nd National Conference on Fluid Power 2011, NCFP, 2011. I11-9.3.

2. Pelosi, M. and M. Ivantysynova, Heat Transfer and Thermal Elastic Deformation Analysis on the Piston:Cylinder Interface of Axial Piston Machines. Journal of Tribology, 2012. 134(041101): p. 1-15.

3. Zecchi, M., A novel fluid structure interaction and thermal model to predict the cylinder block/valve plate interface performance in swash plate type axial piston machines. Dissertation Purdue University, 2013.

4. Zecchi, M. and M. Ivantysynova, Spherical valve plate design in axial piston machines - A novel thermo-elasto-hydrodynamic model to predict the lubricating interface performance. The 8th International Conference on Fluid Power Transmission and Control (ICFP 2013), Apr. 9-11, 2013, Hangzhou, China, 2013: p. 325 - 329.

5. Yamaguchi, A. and M. Tsuchimoto, Bearing Seal Characteristics of the Oil Film between a Valve Plate and a Cylinderblock of Axial Piston Pumps. Hydraulics \& Pneumatics, 1982. 13(1): p. 55-60.

6. Yamaguchi, A., et al., Characteristics of Fluid Films Between a Valve Plate and a Cylinder Block of Axial Piston Pumps and Motors. Hydraulics \& Pneumatics, 1984. 15(4): p. 64-72.
7. Yamaguchi, A., et al., Bearing/Seal Characteristics of the Film between a Valve Plate and a Cylinder Block of Axial Piston Pumps (3rd Report- Effects of Fluid Types and Theoretical Discussions). Hydraulics \& Pneumatics, 1987. 18(7): p. 543-550.

8. Wieczorek, U., Simulation of the gap flow in sealing and bearing gaps of axial piston machines. Proc. of 1st FPNI-PhD Symposium Hamburg, 2000.

9. Wieczorek, U. and M. Ivantysynova, Computer aided optimization of bearing and sealing gaps in hydrostatic machines - the simulation tool CASPAR. International Journal of Fluid Power, 2002. 3(1).

10. Ivantysynova, M., A new approach to the design of sealing and bearing gaps of displacement machines. Proc. 4th Int Symposium on Fluid Power, 1999.

11. Huang, C. and M. Ivantysynova, A new approach to predict the load carrying ability of the gap between valve plate and cylinder block. Proc. Bath Workshop on Power Transmission \& Motion Control, PTMC 2003, Bath, UK, 2003.

12. Ivantysynova, M., Prediction of pump and motor performance by computer simulation. 1st Int. Conf. on Computational Methods in Fluid Power Technology, Melbourne, Australia, 2003, 2003.

13. Achten, P.A.J. and M.P.A. Schellekens, Deformation effects on the load carrying capacity of the barrel bearing in axial piston pumps and motors. Proc. IMECE2006, 2006 ASME International Mechanical Engineering Congress and Expo November 5-10, 2006, Chicago, Illinois, USA, 2006. IMECE2006-13223.

14. Schenk, A., M. Zecchi, and M. Ivantysynova, Accurate Prediction of Axial Piston Machine's Performance Through a Thermo-Elasto-Hydrodynamic Simulation Model. Proc. of the ASME/BATH 2013 Symposium on Fluid Power \& Motion Control, FPMC2013, October 6-9, 2013, Sarasota, Florida, USA, 2013.

15. Chacon, R. and M. Ivantysynova, An investigation of the impact of micro surface on the cylinder block/valve plate interface performance. Proc. of the 8th FPNI Ph.D Symposium on Fluid Power June 11-13, 2014, Lappeenranta, Finland, 2014(FPNI2014-7837-0).

16. Ivantysynova, M. and J. Baker, Power Loss in the Lubricating Gap between Cylinder Block and Valve Plate of Swash Plate Type Axial Piston Machines. International Journal of Fluid Power, 2009. 10(2): p. $29-43$

17. Shin, J.-H. and K.-W. Kim, Effect of surface non-flatness on the lubrication characteristics in the valve part of a swash-plate type axial piston pump. Meccanica, 2014. 49(5): p. 1275-1295. 
18. Bräckelmann, U., Reibung, Steifigkeit und Dämpfung in Schrägscheiben-Axialkolbenpumpen und -motoren. Thesis Ruhr-Universität Bochum, 2006.

19. Bräckelmann, U. and F. Jarchow, Reibung, Steifigkeit und Dämpfung in Schrägscheiben-Axialkolbenpumpen und-motoren. Wissensportal Baumaschine.de, 2006.

20. Pelosi, M., M. Zecchi, and M. Ivantysynova, A fully coupled thermo-elastic model for the rotating kit of axial piston machines. Proc. Bath ASME Symposium on Fluid Power and Motion Control FPMC, 2010: p. $217-234$.

21. Bergada, J.M., J. Watton, and S. Kumar, Pressure, Flow, Force, and Torque Between the Barrel and Port Plate in an Axial Piston Pump. Journal of Dynamic Systems, Measurement, and Control, 2008. 130(1): p. 011011 .

22. Bergada, J.M., et al., Experimental investigation in axial piston pumps barrel dynamics. Proc. Flucome 2009, 10th Int. Conf. on Fluid Control, Measurements, and Visualization, August 17-21, 2009, Moscow, Russia, 2009.

23. Bergada, J.M., et al., The effect of oil pressure and temperature on barrel film thickness and barrel dynamics of an axial piston pump. Meccanica, 2011. 47(3): p. 639-654.

24. Wegner, S., et al., Experimental Investigation of the Cylinder Block Movement in an Axial Piston Machine. Proc. ASME/BATH 2015 Symposium on Fluid Power and Motion Control, FPMC2015, Oct. 12-14, 2015, Chicago, Ill., USA, 2015(FPMC2015-9529).

25. Wegner, S., F. Löschner, and S. Gels, Validation of the physical effect implementation in a simulation model for the cylinder block/valve plate contact supported by experimental investigations. Proc. 10th IFK International Fluid Power Conference, Dresden , Germany, March 8-10, 2016. 1(269-282).

26. Han, L., S. Wang, and C. Zhang, A partial lubrication model between valve plate and cylinder block in axial piston pumps. Proceedings of the Institution of $\mathrm{Me}-$ chanical Engineers, Part C: Journal of Mechanical Engineering Science, 2015. 229(17): p. 3201-3217.

27. Zhang, C., et al., A new dynamic seven-stage model for thickness prediction of the film between valve plate and cylinder block in axial piston pumps. Advances in Mechanical Engineering, 2016. 8(9).

28. Manring, N., Torque on the cylinder block of an axialpiston swash-plate type hydrostatic pump. Retrospective Theses and Dissertations. Paper 11164, 1996.

29. Manring, N.D., Tipping the Cylinder Block of an axialpiston swash-plate type hydrostatic machine. Transactions of the ASME, 2000. 122: p. 216-221.
30. Manring, N.D., et al., Scaling the Speed Limitations for Axial-Piston Swash-Plate Type Hydrostatic Machines. Journal of Dynamic Systems, Measurement, and Control, 2014. 136(3): p. 031004.

31. Jeong, H.-S. and H.-E. Kim, On the instantaneous and average piston friction of swash plate type hydraulic axial piston machines. KSME International Journal, 2004. 18(10): p. 1700-1711.

32. Achten, P., T.v.d. Brink, and M. Schellekens, Design of a variable displacement floating cup pump. Proc. SICFP'05, June 1-3, 2005, Linköping, Sweden, 2005.

33. Achten, P.A.J., T.L.v.d. Brink, and G.E.M. Vael, $A$ robust hydrostatic thrust bearing for hydrostatic machines. Proc. 7.IFK, March 22-24, 2010, Aachen, Germany, 2010: p. 100-112.

34. Achten, P.A.J., T.L.v.d. Brink, and J.W. Potma, Movement of the Cups on the Barrel Plate of a Floating Cup, Axial Piston Machine. Int. Journal of Fluid Power, 2004. 5(2): p. 25-33. 